\title{
GENETIC DIVERSITY, POPULATION STRUCTURE AND EVALUATION OF BREAD WHEAT GENOTYPES UNDER HIGH TEMPERATURE STRESS
}

\author{
R. Farheen ${ }^{1}$, M. Q. Ahmad ${ }^{1 *}$, M. A. Saleem ${ }^{1}$, A. Qayyum ${ }^{1}$, E. Noor ${ }^{1}$, W. Malik ${ }^{1}$, A. Saleem ${ }^{3}$, S. H. Khan ${ }^{2}$, and \\ S. Mahmood ${ }^{1}$
}

${ }^{1}$ Department of Plant Breeding and Genetics, Faculty of Agricultural Sciences and Technology, Bahauddin Zakariya University, Multan; ${ }^{2}$ Center for Advanced Studies in Agriculture and Food Security (CAS-AFS), University of Agriculture, Faisalabad; ${ }^{31}$ Department of Plant Breeding and Genetics, University of Agriculture, Faisalabad. *Corresponding author's emai: mqadirahmad@bzu.edu.pk

\begin{abstract}
High temperature stress at reproductive stage is a prominent factor in various crop plants for yield reduction particularly wheat. In current study the performance of 50 wheat genotypes was analyzed under high temperature stress. To ascertain genetic diversity and population structure, 25 SSR markers were employed. The study was conducted for two consecutive years during 2016-17 and 2017-18 under normal and high temperature stress using randomized complete block design replicated thrice. Significant variation was found among genotypes, treatment and year factors for all the studied traits. Harvest index showed highest value of broad sense heritabilty 95.58. Genotype by trait biplot on the basis of relative performance dataset showed highest variation for yield traits (grains/spike and yield) followed by plant height, peduncle length and days to physiological maturity. The genotypes Yecora-70 and Shafaq-06 appeared tolerant because these genotypes showed less reduction in yield under heat stress. Population structure analysis grouped the genotypes into three sub-populations (A, B and C) which consisted of 15, 18 and 17 genotypes in each subpopulation, respectively. Genetic diversity values were ranged from 0.12 to 0.56 and polymorphism information contents $(P I C)$ value from 0.11 to 0.45 . Overall, the germplasm under investigation revealed low level of genetic polymorphism. Therefore, to combat challenges being posed by climate change wheat germplasm should be enriched by introducing novel genetic resources.
\end{abstract}

Key words: Polymorphism, biplot, heterozygosity, allele, heat stress.

https://doi.org/10.36899/JAPS.2021.4.0298

Published online December 15, 2020

\section{INTRODUCTION}

Wheat (Triticum aestivum L.) is a vital nutritive cereal for human in global food chain (Bonjean and Angus, 2001). It is $3^{\text {rd }}$ leading cereal crop after maize and rice that together produce nearly 57\% world's calories from agricultural source (Tilman et al., 2011). Wheat is a long day and thermo-sensitive plant. The optimum growth temperature required for wheat growth is $25^{\circ} \mathrm{C}$ but it can grow well from minimum $3-4{ }^{\circ} \mathrm{C}$ to maximum $30-32{ }^{\circ} \mathrm{C}$ (Akbar, 2007). Extensive phase of industrialization and urbanization has eroded the environment due to greenhouse gases emission at large scale. As climatic conditions continue to change, the occurrence and severity of abiotic stresses effecting global crop production is becoming severe and resulting in yield reduction in various crop plants including wheat. Results of climatic predictions show that annual global temperature may rise from $1-4{ }^{\circ} \mathrm{C}$ by the end of this century (Driedonks et al., 2016).

Many wheat researchers have reported effects of high temperature stress on morphological and physiological processes at vegetative and reproductive phases (Cossani and Reynolds, 2012; Feng et al., 2014; Chaturvedi et al., 2017). In many wheat producing countries including Pakistan, India, Australia, United States and Mexico, high temperature stress at grain filling period showed adverse effects on yield and quality of grain (Bonnet et al., 2008).

High temperature stress may reduce wheat yield $33.6 \%$ to $50 \%$ (Chatrath et al., 2007; Joshi et al., 2007). Reduction in yield due to high temperature stress is not only limited to wheat, it is also effecting other major crops i.e., sorghum, rice and maize (Stratonovitch and Semenov, 2015; Prasad et al., 2008; Yang et al., 2015; Shi et al., 2016). High temperature at reproductive stage may cause pollen abortion, premature senescence and shrinked kernels resulting in poor kernel quality and yield reduction (Xu et al., 2000a; Jiang et al., 2004). Yield potential of wheat cannot be achieved under heat stressed conditions. High yield of wheat is very crucial to feed future generations.

Heat stress is a complex trait that is determined by several genes. For tolerant genotype selection, precise phenotyping in multi-year trials to account genotype by environment interaction should be conducted. Genetic variation is a pre-requisite for the identification of heat tolerant genotypes because it has potential to deal with multiple stresses. Overall a reduction in the genetic diversity of major crops particularly in wheat has been 
observed due to domestication and extensive breeding efforts.

Presence of genetic diversity increases the chances of development of a superior line. For the proper utilization, extent and distribution of genetic diversity should be determined. Some researchers depicted diversity using morphological characters (Takumi et al., 2009). But due to environmental effects and changes during various growth stages, reliability only on morphological traits cannot be guaranteed particularly in similar populations. Therefore, for precise estimates of genetic diversity, use of molecular markers particularly simple sequence repeats (SSR) have proved beneficial (Ahmad et al., 2014, Sajjad et al., 2014, Ahmad et al., 2015).

Genetic diversity is very crucial for survivability and adaptability of wheat against high temperature stress. For selecting parents having heat tolerance, the patterns of genetic diversity should be estimated. Genetic diversity and population structure are useful for determination of nature of population and molecular breeding. Many studies have shown the importance of genetic diversity and population structure for broadening germplasm genetic base through precise selection of parents (Zhang et al., 2011; Abebe and Leon, 2012). Therefore, understanding of population structure and genetic diversity is key for selection and further improvement (Ahmad et al., 2014). Considering subgroups within a population exclude the biasness of results due to human driven selection, genetic drift and geographical origin (Flint-Garcia, 2003; Buckler and Thornsberry, 2002).

Keeping in view the drastic effects of high temperature stress on wheat yield following study was undertaken to explore performance of Pakistani wheat material under high temperature stress. Furthermore, population structure and genetic diversity analysis were carried out to know the genetic relatedness and differences among these genotypes.

\section{MATERIALS AND METHODS}

Plant material and evaluation of genotypes under field conditions for high temperature stress tolerance: Fifty wheat genotypes developed in Pakistan from the course of time were included in the study (Table 1). Genotypes were included on the basis of their performance and the study was executed at research area of department of Plant Breeding and Genetics, Bahauddin Zakariya University, Multan. Plant material was evaluated for two consecutive years during 2016-2017 and 2017-2018 under high temperature stress and non-stressed conditions. Field was prepared following recommended agronomic practices. The trial under normal condition was sown on November 15, during 2016-2018 whereas; to develop heat stress the sowing was delayed till $30^{\text {th }}$ of December. Average temperature and rainfall during both years was recorded (Fig. 1). The plant material was sown in three replications using randomized complete block design (RCBD). Each entry was planted on $450 \mathrm{~cm}$ long row using hand drill. Distance between rows and plants was maintained $22.5 \mathrm{~cm}$ and $12.5 \mathrm{~cm}$, respectively. After germination of seeds, plant to plant distance was maintained by thinning. Recommended agronomic practices were followed to raise healthy crop.

Genotypes were characterized for various morphological traits under both conditions i.e. yield $(\mathrm{Y})$, grains/spike (GPS), 1000-grain weight (TGW), Individual spike weight (ISW), days to physiological maturity (DPM), canopy temperature (CT), days to heading (DTH), flag leaf length (FLL), flag leaf width (FLW), peduncle length (PDL), awn length (AL) and plant height (PH). For height and related traits, measurements were carried out in the field using measuring scale at maturity. Yield related traits were determined after harvesting using digital electric balance. Canopy temperature was recorded before and after heading using digital canopy meter.

Nucleic acid isolation and SSR genotyping: Cetyl trimethyl ammonium bromide (CTAB) method was followed to extract the DNA from young fresh leaves of wheat (Doyle and Doyle, 1990). The DNA samples were genotyped with 25 SSR markers. Polymerase chain reaction (PCR) was performed for $20 \mu \mathrm{l}$ reaction mixture containing $2 \mu \mathrm{l}$ of $10 \mathrm{x}$ buffer, $2.5 \mu \mathrm{l}$ of $\mathrm{Mgcl}_{2}, 1 \mu \mathrm{l}$ each of Forward and reverse primer $(10 \mu \mathrm{M}), 1 \mu \mathrm{l}$ of dNTPs mix $(10 \mathrm{mM}), 11.4 \mu \mathrm{l}$ of $\mathrm{ddH}_{2} \mathrm{O}, 0.1 \mathrm{ul}$ of Taq polymerase and 1 $\mu 1$ DNA (30ng/ $\mu \mathrm{l})$. The PCR analysis were conducted on thermal cycler (BIORAD-C1000). Following thermal conditions were used for amplification of DNA, initial denaturation at $94^{\circ} \mathrm{C}$ for 4 minutes, 35 cycles of denaturation at $94^{\circ} \mathrm{C}$ for 1 minute, 1 minute annealing at $55^{\circ} \mathrm{C}$ (Primer specific) and 1 minute extension at $72^{\circ} \mathrm{C}$. After PCR reaction, 8ul of PCR product was loaded on $2.5 \%$ agarose gel $(0.8 \mu \mathrm{g} / \mathrm{ml})$. A size specific, $50 \mathrm{bp}$ DNA ladder was used to determine exact PCR fragment size. Gel was run for 2 hours at 80 volts $(2.5 \mathrm{~V} / \mathrm{cm})$ and photographed by Gel-Document System.

Statistical analysis: Analysis of variance was performed to check variation among genotypes at 5\% probability level (Steel et al., 1997). For this purpose, a Two-way ANOVA was constructed.

Principal component analysis and genotype by trait biplot: To determine the extent of diversity in the expression of various plant traits, PCA was performed (Swan et al., 1995). PCA was performed on the basis of relative performance dataset. Relative performance dataset was obtained by following the method adopted by (Ahmad et al., 2014). Same dataset was used to execute genotype by trait biplot to identify stress tolerance genotypes.

Structure analysis using population structure: Population structure analysis based on polymorphic SSR data was performed using software STRUCTURE 
(Pritchard et al., 2000). To optimize likelihood number for sub-populations, the STRUCTURE was run with 30,000 MCMC repetitions and 30000 burn in period of 2-20 subpopulation's range. The peak for sub-populations was identified at 3 likelihood. Keeping k-value at 3, the STRUTURE was again applied for a period of 100,000 MCMC repetitions and 10000 burns in iteration to divide the genotypes into 3 sub-populations (Pritchard et al., 2000).

Analysis for genetic diversity, heterozygosity and polymorphism information content: Power marker software was used to determine various parameters of genetic diversity, number of alleles, heterozygosity and polymorphism information content (PIC) (Liu and Muse, 2005).

\section{RESULTS}

Phenotypic evaluation and analysis of variance: Fifty genotypes of wheat were evaluated under stressed and non-stressed conditions. High temperature stress at reproductive stage was applied by delayed sowing. Maximum and minimum performance for the studied traits under high temperature stress and normal conditions during 2016-17 and 2017-18 are listed in Table 2 and 3, respectively. For the first year, AAS-11 appeared to be the best performer with $84 \mathrm{~g}$ yield per plant followed by K-65 with $74 \mathrm{~g}$ yield per plant. Whereas under high temperature stress the genotypes Yecora-70 and Shafaq-06 performed better ( $45 \mathrm{~g}$ yield per plant) than all the other studied genotypes. During year 2017-18 best performing genotypes were AAS-11 and Chakwal-86. AAS-11 performed best under normal conditions during both years but under high temperature stress major reduction in yield/plant was observed. For DTH and DPM, effect of high temperature stress during 2017-18 was more prevalent (Table 3). Mean days to heading were from 99.7 days and 77.4 days under normal and stress conditions, respectively. Similarly for days to physiological maturity, mean performance was observed as 132.7 days under normal conditions and 103.3 days under high temperature stress conditions.

To check the significance of observed variation, ANOVA was performed that depicted significant results for studied characters i.e. DTH, DPM, PDL, PH, FLW, FLL, AL, HI, TGW, ISW, Yield and GPS except CT. Year and Treatment factors also revealed significant amount of variation among studied traits. Two way interactions $\left(G^{*} T\right)$ and $\left(T^{*} Y\right)$ were also significant (Table 4).

Principal component analysis based on relative performance of genotype: Relative performance or stress tolerance index value of each genotype was calculated following Ahmad et al., (2014) and used for determination of tolerant wheat genotypes. Principle component analysis was performed using stress tolerance index values (Table
5 and 6). For the year 2016-17, seventeen PCs were executed, out of which five PCs showed $67.867 \%$ cumulative variation (Table 5). Eigen values of 5PCs were observed as 2.167, 1.923, 1.825, 1.778 and 1.130, respectively (Table 5).

In the $1^{\text {st }} \mathrm{PCA}$, more variation for stress tolerance index was observed for yield related traits i.e. yield (0.537) and GPS (0.496) whereas; PC 2 was more related to DPM (0.301), FLW (0.349) and AL (0.32). PC3 showed more variation for DTH (0.411), PDL (0.483) and TGW (0.262). During the year 2017-2018, five PCs illustrated more than one value and accounted $70.089 \%$ cumulative variation as shown in Table 6. Eigen values of 5 PCs were observed as $3.245,1.938,1.524,1.364$ and 1.040, respectively (Table 6). Higher loading values were observed for plant developmental traits in PC1 i.e. PL (0.648), PH (0.365), FLL, (0.603), DPM (0.416). PC 2 was more related to DTH (0.395), Yield (0.325) and GPS (0.272). PC3 showed more variation for $\mathrm{CT}$ and $\mathrm{AL}$. In PC4, maximum loading values were shown by $\mathrm{HI}$ and TGW. PC5 showed maximum loading value for FLW.

Genotype-by-trait biplot analysis: Length and angle between the trait vectors was used as a parameter for selection of genotypes. The trait vectors showed less angle were having more resemblance as compared to the trait separated with great angle. Similarly, the traits which showed more vector length were more diverse in relation to the traits showing short vector length.

On the basis of relative performance dataset (2016-2017) the diverse genotypes were placed on the polygon vertex which include G3, G27, G7, G2 and G40. Among traits maximum variation in response to high temperature stress was depicted by grains/spike and yield followed by peduncle length, plant height and days to physiological maturity. The heat stress affected most of the yield traits in similar manner (Fig. 2). Most of the yield traits (yield, individual spike weight, grains/spike) run parallel on biplot. Similarly, height related traits were also placed parallel to each other on the polygon (Fig. 2) which showed their strong association with each other. Days to heading vector was placed opposite to yield related traits. On the basis of relative performance, K-65, AAS-11 and Mexipak appeared as stress susceptible genotypes. Although under normal conditions these genotypes were high yielding but due to elevated temperature at grain formation stage they could not maintain their yield. The genotypes which appeared as the tolerant genotypes include Yecora-70 and Shafaq-06. These genotypes showed less reduction in yield under heat stress.

For the year 2017-2018, the genotypes G36, G21, G9, G13, G48 and G3 were appeared as most diverse. Maximum variation in response to high temperature stress was shown by peduncle length followed by DPM and DTH. Yield related traits showed less variation as compared to previous year. Harvest index was placed 
opposite to other yield related traits (Fig. 3). For the year 2017-18, genotypes PB-85 and C-591 showed maximum reduction in yield and Potohar in grains/spike. AAS-11 and Inqlab-91 showed highest reduction for DTH and DPM.

Population structure analysis: Genetic resemblance nature among studied entries was determined by STRUCTURE analysis. The whole population was subdivided into three (A, B and C) sub-populations (Fig. 4 and 5). These sub-populations were consisted of 15, 18 and 17 genotypes, respectively. First values for differences among populations were observed significant.

Structure analysis and tolerance within subpopulations: As structure analysis was performed using molecular dataset and relative performance was obtained from phenotypic dataset some interesting similarities were observed among grouping of genotypes. The genotypes LYP-73, T11, Pari-73 and Barani-83 were placed in same sub-population C. On relative performance dataset during 2017-18, they were placed very close to each other in the same quadrant. Whereas, during 2016-17 genotypes Pari-73 and LYP-73 were placed in the same quadrant which showed high genetic resemblance between these two genotypes. Similarly genotypes Darabi-11, Pak81 and Mexipak were place in the same subpopulation A and these genotypes were also located in close proximity on relative performance dataset.

Genetic diversity analysis: Out of 25 SSR markers, 15 SSRs showed polymorphism which were used for further study. Fifty wheat genotypes scanned with 15 polymorphic SSRs provided a total of 33 alleles with 2.2 average alleles/marker. Number of alleles ranged from two to three. Maximum polymorphic alleles were showed by SSR markers WMC 332, WMC434 and WMC70 (Table 7). Major allele frequency was from 0.479 (WMC 332) to 0.937 (WMC 289) with average value of 0.749 alleles. Values of PIC ranged from 0.110 to 0.458 with 0.28 average value. Gene diversity was observed from 0.117 to 0.557 with 0.338 average value. The heterozygosity level ranged from 0 to 0.021 (Table 7).

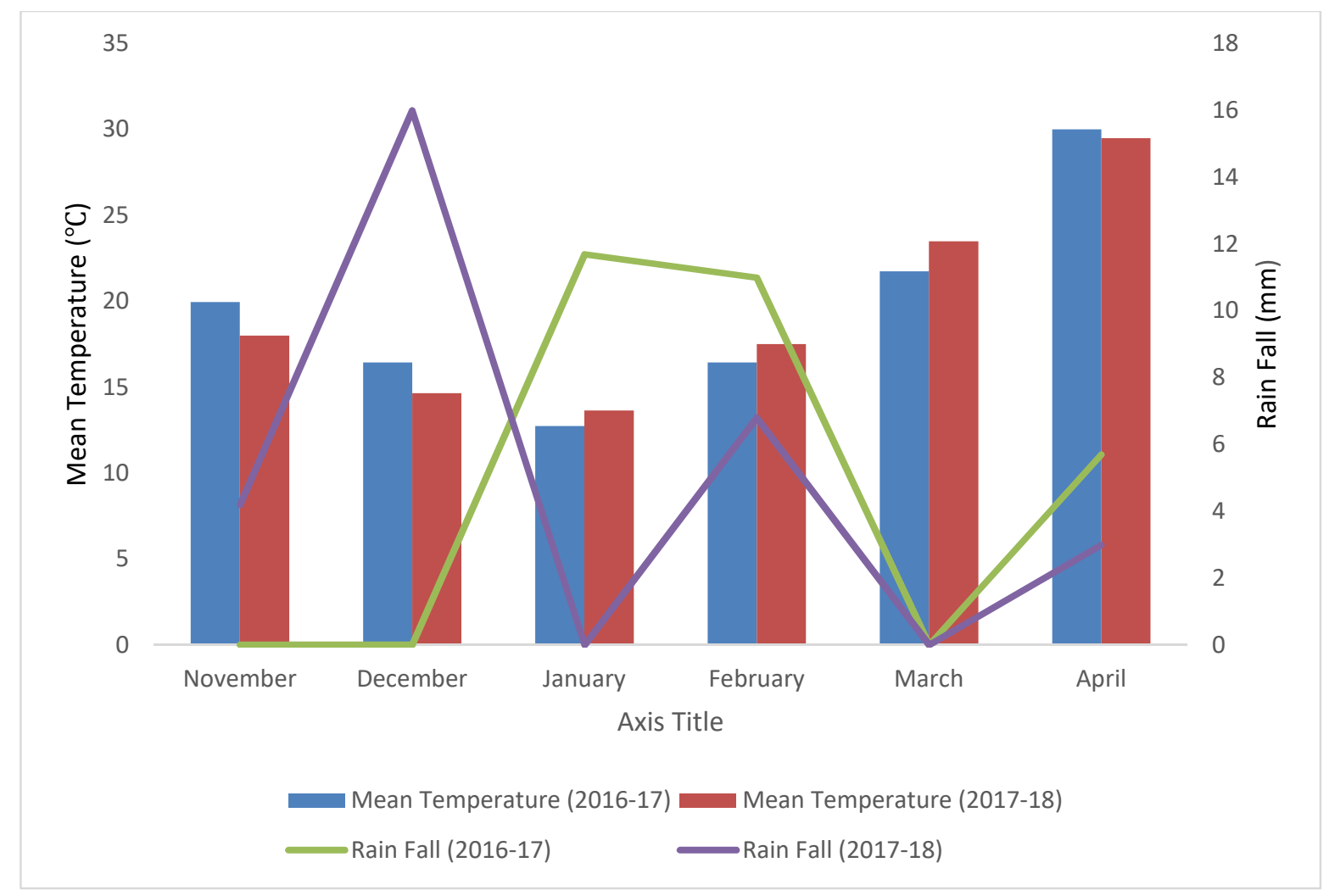

Fig.1 Average temperature and rainfall during 2016-18. 


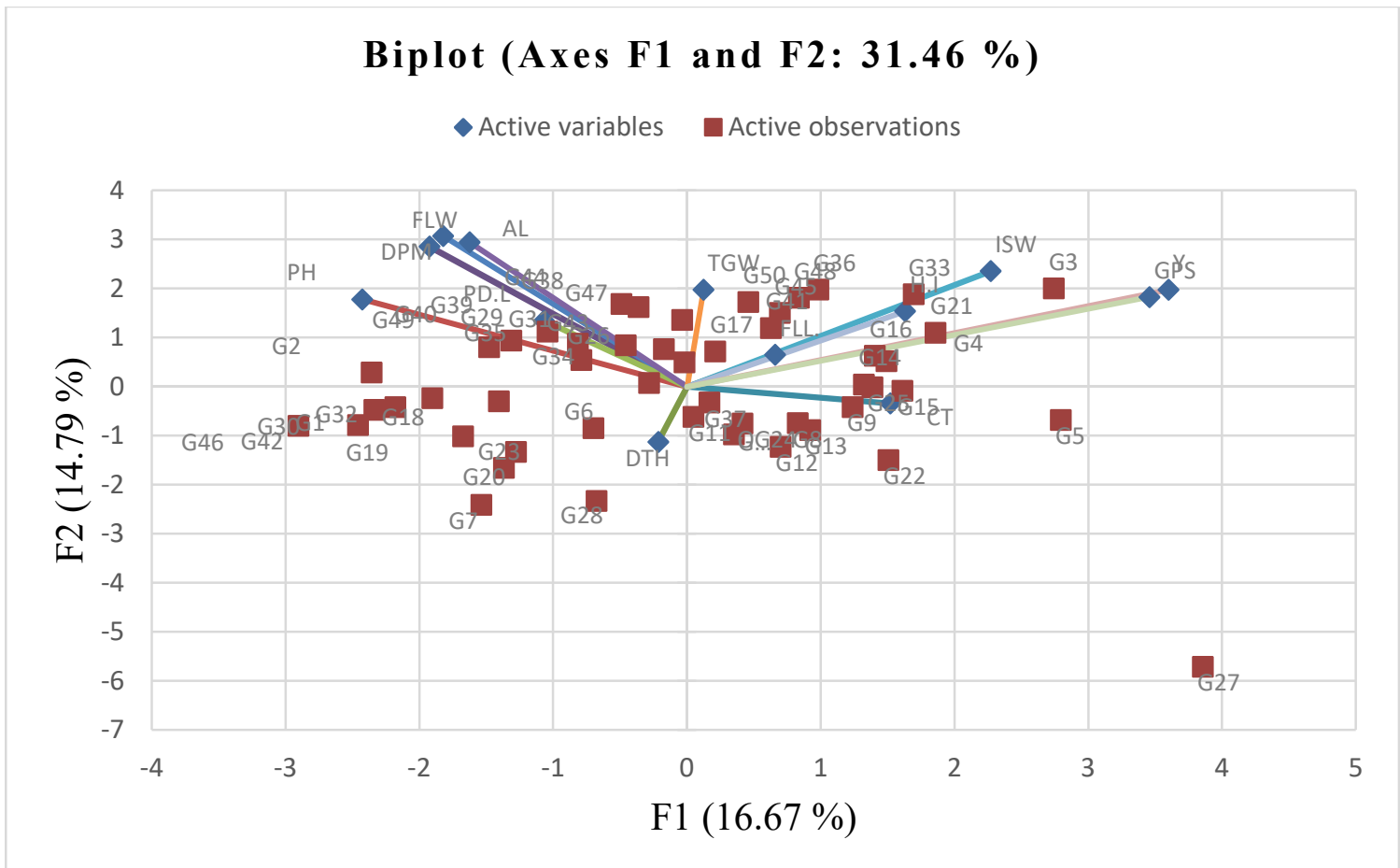

Fig 2. Genotype-by-trait biplot based on relative performance dataset (2016-2017)

$\mathrm{DTH}=$ Days to heading, DPM= Days to physiological maturity, CT= Canopy temperature, FLL= Flag leaf length, FLW= Flag leaf width, $\mathrm{PH}=$ Plant height, $\mathrm{PDL}=$ Peduncle length, $\mathrm{AL}=$ Awn length, $\mathrm{TGW}=1000$-grain weight, $\mathrm{ISW}=\mathrm{Individual}$ spike weight, $\mathrm{HI}=$ Harvest index, GPS= Grain/spike, Y= Yield

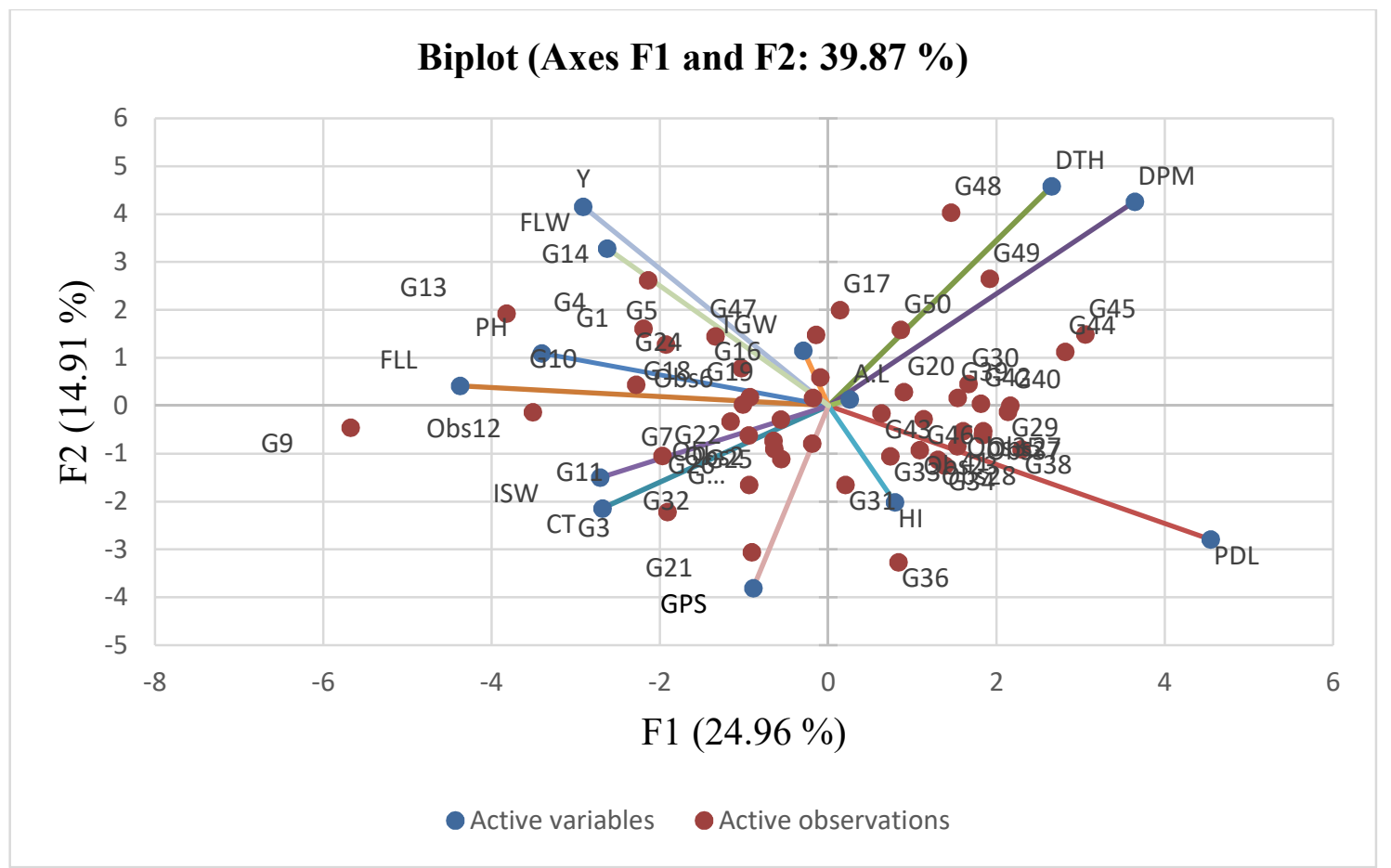

Fig. 3: Genotype-by-trait biplot based on relative performance dataset (2017-2018).

$\mathrm{DTH}=$ Days to heading, DPM= Days to physiological maturity, CT= Canopy temperature, FLL= Flag leaf length, FLW= Flag leaf width, $\mathrm{PH}=$ Plant height, $\mathrm{PDL}=$ Peduncle length, $\mathrm{AL}=$ Awn length, $\mathrm{TGW}=1000$-grain weight, $\mathrm{ISW}=\mathrm{Individual}$ spike weight, $\mathrm{HI}=$ Harvest index, GPS $=$ Grain/spike, $Y=$ Yield 


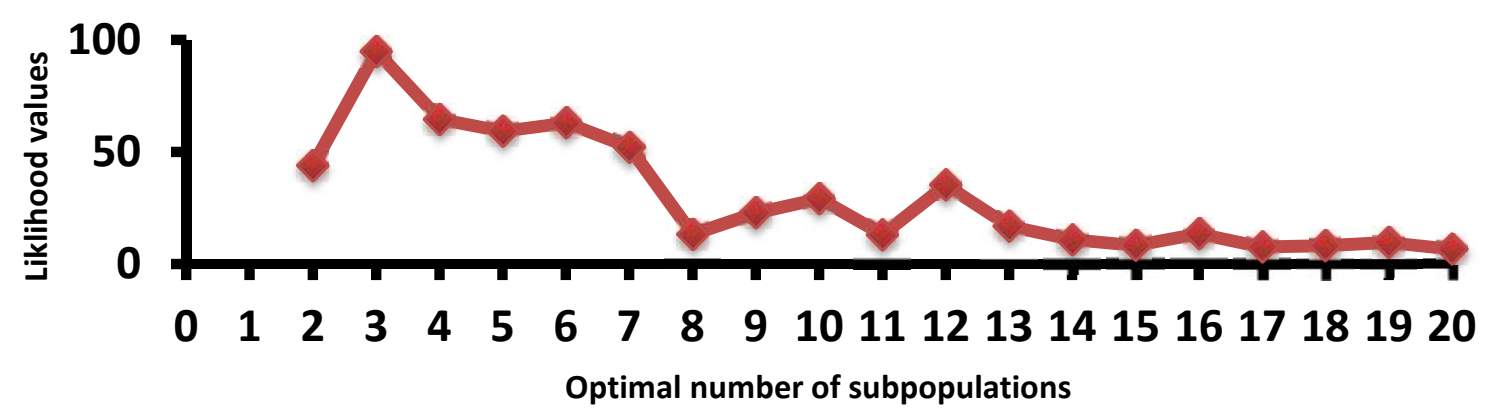

Fig. 4. Identification of optimal number of subpopulations when $K$ ranged from 2 to 20 and revealed the peak at 3.

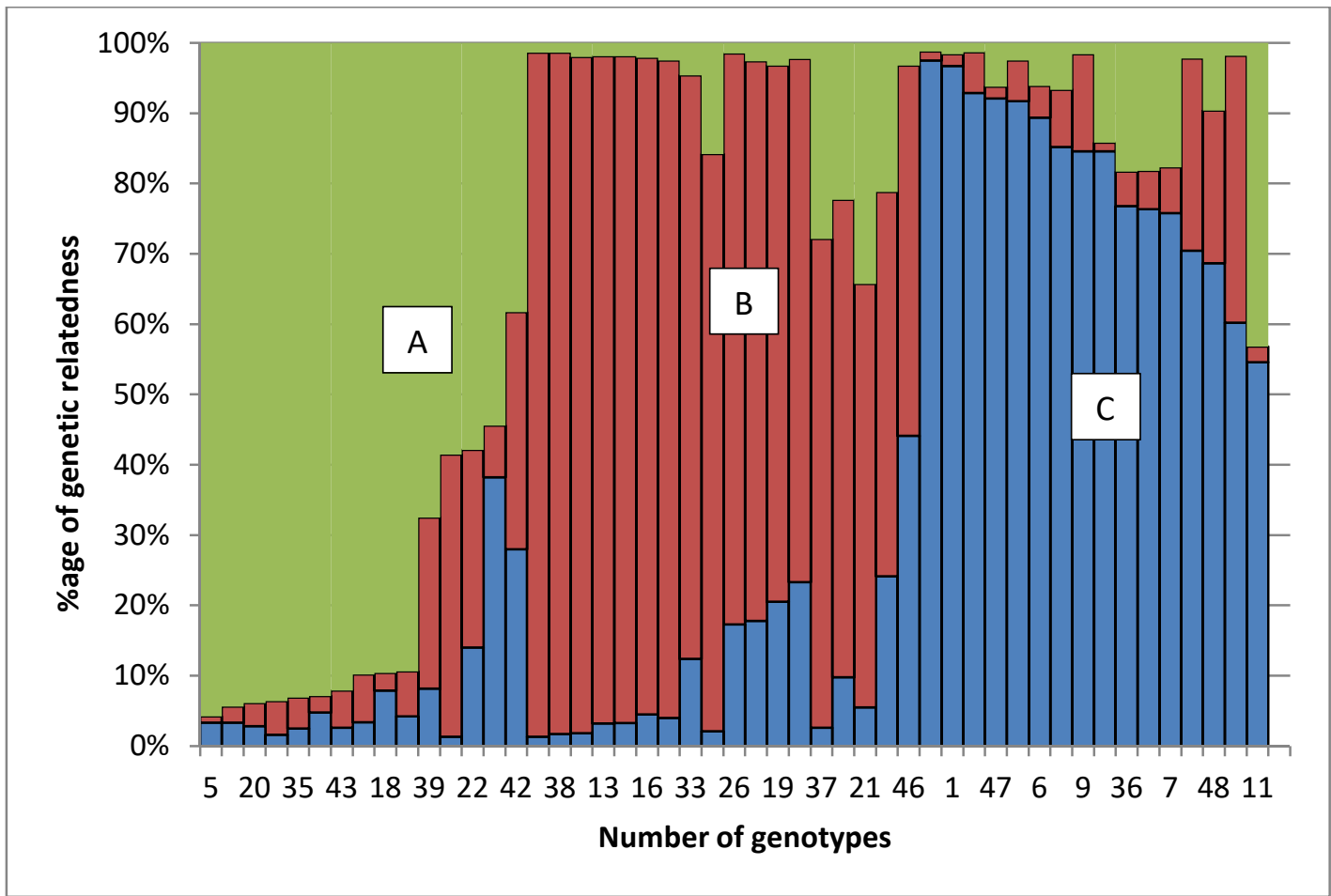

Fig. 5. Population structure revealed by STRUCTURE on the basis of SSRs.

Table 1. Numbers and Name of wheat genotypes used in this study.

\begin{tabular}{cccc}
\hline Numbers & Name of genotypes & Numbers & Name of genotypes \\
\hline 1 & Yecora-70 & 26 & WL-711 \\
2 & Kohinoor-83 & 27 & Barani-70 \\
3 & Mexipak & 28 & Barani-83 \\
4 & Chakwal-86 & 29 & Pari-73 \\
5 & SA-75 & 30 & Shafaq-06 \\
6 & T-9 & 31 & LYP-73 \\
7 & LU-26S & 32 & Seher-06 \\
8 & C-273 & 33 & K-65 \\
9 & PB-76 & 34 & C-217 \\
10 & FSD-83 & 35 & Miraj-08 \\
11 & C-250 & 36 & T-11 \\
12 & Pak-81 & 37 & Rohtas-90 \\
13 & C-591 & 38 & Blue Silver \\
14 & PB-85 & 39 & Parwaz-94 \\
\hline
\end{tabular}




\begin{tabular}{lccc}
\hline 15 & C-220 & 40 & D-97 \\
16 & SA-42 & 41 & Dirk \\
17 & Chakwal-50 & 42 & Galaxy-13 \\
18 & FSD-85 & 43 & Ujala-16 \\
19 & Sandal-73 & 44 & Lasani-08 \\
20 & PB-96 & 45 & Millat-11 \\
21 & Potohar & 46 & Punjab-11 \\
22 & Darabi-11 & 47 & Wadank-85 \\
23 & Chenab-70 & 48 & AAS-11 \\
24 & Shahkar-95 & 49 & Inqlab-91 \\
25 & Pasban-90 & 50 & PB-81 \\
\hline
\end{tabular}

Table 2. Mean performance and range of the genotypes for studied traits during (2016-17).

\begin{tabular}{|c|c|c|c|c|c|c|}
\hline \multirow[t]{3}{*}{ Traits } & \multicolumn{3}{|c|}{ Normal } & \multicolumn{3}{|c|}{ Heat } \\
\hline & \multirow[t]{2}{*}{ Mean } & \multicolumn{2}{|c|}{ Range } & \multirow[t]{2}{*}{ Mean } & \multicolumn{2}{|c|}{ Range } \\
\hline & & Min. & Max. & & Min. & Max. \\
\hline Days to heading & 92.43 & 82 & 111 & 87.15 & 78 & 108 \\
\hline Canopy temperature $\left({ }^{\circ} \mathrm{C}\right)$ & 25.21 & 21.9 & 27.65 & 24.89 & 21 & 26.1 \\
\hline Flag leaf length $(\mathrm{cm})$ & 23.86 & 19.2 & 26.40 & 20.03 & 13.2 & 24.14 \\
\hline Flag leaf width $(\mathrm{cm})$ & 01.70 & 1.06 & 2 & 1.59 & 1.2 & 1.3 \\
\hline Days to physiological maturity & 133.90 & 111 & 130 & 117.87 & 109 & 127 \\
\hline Plant height $(\mathrm{cm})$ & 95.70 & 72.2 & 129.40 & 83.65 & 70.05 & 129.4 \\
\hline Peduncle length $(\mathrm{cm})$ & 41.96 & 28.4 & 57 & 33.21 & 23.44 & 49 \\
\hline Awn length $(\mathrm{cm})$ & 6.26 & 1.1 & 8 & 5.36 & 1.1 & 7.16 \\
\hline Individual spike weight $(\mathrm{g})$ & 2.70 & 1.67 & 3.49 & 1.60 & 0.8 & 2.6 \\
\hline 1000 grain weight $(\mathrm{g})$ & 49.52 & 43.53 & 62.70 & 44.86 & 37.73 & 50.73 \\
\hline Grains per spike & 46.90 & 38.6 & 59.82 & 36.5 & 26.3 & 45.7 \\
\hline Yield (g) & 46.26 & 38 & 49 & 42.18 & 37 & 47 \\
\hline
\end{tabular}

Table 3. Mean performance and range of the genotypes for studied traits during (2017-18).

\begin{tabular}{|c|c|c|c|c|c|c|}
\hline \multirow{3}{*}{ Traits } & \multicolumn{3}{|c|}{ Normal } & \multicolumn{3}{|c|}{ Heat } \\
\hline & \multirow[t]{2}{*}{ Mean } & \multicolumn{2}{|c|}{ Range } & \multirow[t]{2}{*}{ Mean } & \multicolumn{2}{|c|}{ Range } \\
\hline & & Min. & Max. & & Min. & Max. \\
\hline Days to heading & 99.77 & 90 & 117 & 77.47 & 72 & 90 \\
\hline Canopy temperature $\left({ }^{\circ} \mathrm{C}\right)$ & 27.69 & 28.8 & 24.8 & 25.92 & 23.3 & 27.2 \\
\hline Flag leaf length $(\mathrm{cm})$ & 22.85 & 14.7 & 31.04 & 20.37 & 11.2 & 26.2 \\
\hline Flag leaf width $(\mathrm{cm})$ & 1.83 & 1.3 & 2 & 1.15 & 0.52 & 1.68 \\
\hline Days to physiological maturity & 132.70 & 127 & 137.33 & 103.32 & 97.33 & 112 \\
\hline Plant height $(\mathrm{cm})$ & 89.48 & 67.3 & 122 & 71.19 & 55.76 & 98.52 \\
\hline Peduncle length $(\mathrm{cm})$ & 33.92 & 27.7 & 41.78 & 29.42 & 22.8 & 37.4 \\
\hline Awn length $(\mathrm{cm})$ & 6.23 & 3.82 & 14.1 & 5.04 & 1.26 & 7.26 \\
\hline Individual spike weight (g) & 2.23 & 1.6 & 2 & 1.420 & 0.8 & 2 \\
\hline 1000 grain weight $(\mathrm{g})$ & 49.49 & 42 & 56.66 & 55.853 & 42 & 67.66 \\
\hline Grains per spike & 62.33 & 43.33 & 76.66 & 42.2 & 33.33 & 53.33 \\
\hline Yield (g) & 33.71 & 32 & 40 & 28.266 & 24 & 32 \\
\hline
\end{tabular}


Table 4: Mean squares and broad sense heritabilty values of all the studied traits.

\begin{tabular}{|c|c|c|c|c|c|c|c|c|c|c|c|c|c|c|}
\hline SOV & DF & DTH & DPM & CT & FLL & FLW & PH & PDL & $\mathbf{A L}$ & ISW & TGW & HI & $Y$ & GPS \\
\hline Gen. & 49 & $45.8^{* *}$ & $628^{*}$ & 8 & $26.64^{* *}$ & $48.46^{* *}$ & $545.5^{* *}$ & $140.9^{* *}$ & $4.5^{* *}$ & $158.4^{*}$ & $128.9^{* *}$ & $0.11^{* *}$ & $327.3^{* *}$ & $90.9^{* *}$ \\
\hline Rep. & 2 & $25.9^{*}$ & $1732.3^{* *}$ & $9602.3^{* *}$ & $16.539^{*}$ & $44.69^{*}$ & $42.2^{* *}$ & $5667.7^{* *}$ & $14198^{* *}$ & $621.7^{*}$ & $72.8^{*}$ & $0.002^{*}$ & $151.5^{* *}$ & $198.7^{* *}$ \\
\hline Treat & 1 & $26220^{* *}$ & $76188^{* *}$ & $4116.8^{* *}$ & $533.6^{* *}$ & $3.827^{*}$ & $34042^{* *}$ & $9885.3^{* *}$ & $1704^{* *}$ & $140.1^{*}$ & $22573^{* *}$ & $17.6^{* *}$ & $595.2^{* *}$ & $494.2^{* *}$ \\
\hline $\mathbf{Y}$ & 1 & $411.9^{*}$ & $928.36^{* *}$ & $313.05^{* *}$ & $47.57^{* *}$ & $72.052^{*}$ & $1261.3^{* *}$ & $160.9^{* *}$ & $1436.6^{* *}$ & $123.3^{*}$ & $3908.7^{* *}$ & $21^{* *}$ & $1013^{* *}$ & $177.4^{* *}$ \\
\hline$G^{*} T$ & 49 & $16.2^{*}$ & $56.7^{*}$ & 6 & $17.42^{*}$ & $49.21^{*}$ & $155.1^{*}$ & $80.8^{* *}$ & $2.3^{*}$ & $15841^{*}$ & $82.6^{*}$ & $0.042^{*}$ & $308.5^{*}$ & $1.04^{*}$ \\
\hline $\mathbf{T}^{*} \mathbf{Y}$ & 1 & $11854^{* *}$ & $67.59^{* *}$ & $335.04^{* *}$ & $36.34^{*}$ & $102.6^{* *}$ & $1451.9^{* *}$ & $42071^{* *}$ & $13455^{* *}$ & $983.6^{*}$ & $8966.1^{* *}$ & $32.3^{* *}$ & $13.5^{* *}$ & $993.6^{* *}$ \\
\hline $\mathbf{E}$ & 494 & 18.9 & 55.4 & 118.3 & 22.67 & 48.54 & 159.2 & 143.4 & 170.4 & 114.39 & 56.2 & 0.005 & 290.8 & 496.1 \\
\hline \multicolumn{2}{|c|}{ CV } & 10.946 & 11.615 & 10.4 & 17.51 & 15 & 14.7 & 12.78 & 18.9 & 15 & 14.99 & 18.5 & 15.12 & 14.9 \\
\hline
\end{tabular}

$=$ Significant at $<0.05,{ }^{* *}=$ Highly Significant at $<0.01, \mathrm{SOV}=$ Source of variance, Rep. $=$ Replication, Gen.= Genotype, Treat. $=$ Treatment, $\mathrm{E}=\mathrm{Error}, \mathrm{DF}=\mathrm{Degree}$ of freedom, $\mathrm{Y}=$

Year , DTH= Days to heading, DPM= Days to physiological maturity, CT= Canopy temperature, FLL= Flag leaf length, FLW= Flag leaf width, PH= Plant height, PDL= Peduncle length, $\mathrm{AL}=\mathrm{Awn}$ length, $\mathrm{TGW}=1000$-grain weight, $\mathrm{ISW}=$ Individual spike weight, $\mathrm{HI}=$ Harvest index, GPS= Grain/spike, $\mathrm{Y}=\mathrm{Yiel}$, $\mathrm{CV}=\mathrm{Coefficient}$ of variance. 
Table 5. Principal Component analysis based on relative performance dataset (2016-2017).

\begin{tabular}{cccccc}
\hline Traits & PC-1 & PC-2 & PC-3 & PC-4 & PC-5 \\
\hline DTH & 0.002 & 0.047 & $\mathbf{0 . 4 1 1}$ & 0.008 & 0.050 \\
DPM & 0.154 & $\mathbf{0 . 3 0 1}$ & 0.044 & 0.230 & 0.009 \\
CT & 0.095 & 0.004 & 0.048 & $\mathbf{0 . 3 9 8}$ & 0.021 \\
FLL & 0.018 & 0.016 & 0.092 & 0.074 & $\mathbf{0 . 4 8 1}$ \\
FLW & 0.138 & $\mathbf{0 . 3 4 9}$ & 0.128 & 0.290 & 0.001 \\
PH & 0.245 & 0.117 & 0.001 & $\mathbf{0 . 3 3 9}$ & 0.000 \\
PDL & 0.047 & 0.067 & $\mathbf{0 . 4 8 3}$ & 0.001 & 0.059 \\
AL & 0.110 & $\mathbf{0 . 3 2 0}$ & 0.222 & 0.268 & 0.008 \\
ISW & 0.214 & 0.205 & 0.012 & 0.019 & 0.152 \\
TGW & 0.001 & 0.144 & $\mathbf{0 . 2 6 2}$ & 0.060 & 0.173 \\
HI & 0.110 & 0.087 & 0.100 & 0.065 & 0.083 \\
Y & $\mathbf{0 . 5 3 7}$ & 0.144 & 0.000 & 0.000 & 0.022 \\
GPS & $\mathbf{0 . 4 9 6}$ & 0.123 & 0.021 & 0.026 & 0.071 \\
Eigen value & 2.167 & 1.923 & 1.825 & 1.778 & 1.130 \\
Variability (\%) & 16.667 & 14.790 & 14.042 & 13.676 & 8.693 \\
Cumulative \% & 16.667 & 31.457 & 45.499 & 59.174 & 67.867 \\
\hline DTH
\end{tabular}

$\mathrm{DTH}=$ Days to heading, DPM= Days to physiological maturity, $\mathrm{CT}=$ Canopy temperature, $\mathrm{FLL}=\mathrm{Flag}$ leaf length, $\mathrm{FLW}=\mathrm{Flag}$ leaf width, $\mathrm{PH}=$ Plant height, $\mathrm{PDL}=$ Peduncle length, $\mathrm{AL}=$ Awn length, $\mathrm{TGW}=1000$-grain weight, $\mathrm{ISW}=\mathrm{Individual}$ spike weight, $\mathrm{HI}=$ Harvest index, GPS $=$ Grain/spike, $Y=$ Yield

Note: Values in bold correspond to highest contribution variable.

Table 6. Principal component analysis based on relative performance dataset (2017-2018).

\begin{tabular}{cccccc}
\hline Traits & PC1 & PC2 & PC3 & PC4 & PC5 \\
\hline DTH & 0.221 & $\mathbf{0 . 3 9 5}$ & 0.167 & 0.034 & 0.060 \\
DPM & $\mathbf{0 . 4 1 6}$ & 0.341 & 0.117 & 0.042 & 0.012 \\
CT & 0.227 & 0.086 & $\mathbf{0 . 4 5 3}$ & 0.000 & 0.000 \\
FLL & $\mathbf{0 . 6 0 3}$ & 0.003 & 0.000 & 0.001 & 0.082 \\
FLW & 0.217 & 0.203 & 0.025 & 0.075 & $\mathbf{0 . 2 1 7}$ \\
PH & $\mathbf{0 . 3 6 5}$ & 0.023 & 0.069 & 0.001 & 0.246 \\
PDL & $\mathbf{0 . 6 4 8}$ & 0.146 & 0.041 & 0.011 & 0.005 \\
AL & 0.002 & 0.000 & $\mathbf{0 . 3 5 8}$ & 0.004 & 0.237 \\
ISW & 0.231 & 0.042 & 0.042 & 0.157 & 0.004 \\
HI & 0.019 & 0.076 & 0.009 & $\mathbf{0 . 4 8 5}$ & 0.000 \\
TGW & 0.003 & 0.025 & 0.047 & $\mathbf{0 . 5 0 0}$ & 0.000 \\
Y & 0.267 & $\mathbf{0 . 3 2 5}$ & 0.018 & 0.005 & 0.137 \\
GPS & 0.025 & $\mathbf{0 . 2 7 2}$ & 0.181 & 0.049 & 0.040 \\
Eigen value & 3.245 & 1.938 & 1.524 & 1.364 & 1.040 \\
Variability $(\%)$ & 24.963 & 14.908 & 11.726 & 10.489 & 8.003 \\
Cumulative \% & 24.963 & 39.871 & 51.597 & 62.086 & 70.089 \\
\hline
\end{tabular}

$\mathrm{DTH}=$ Days to heading, DPM= Days to physiological maturity, $\mathrm{CT}=$ Canopy temperature, $\mathrm{FLL}=$ Flag leaf length, FLW= Flag leaf width, $\mathrm{PH}=$ Plant height, $\mathrm{PDL}=$ Peduncle length, $\mathrm{AL}=\mathrm{Awn}$ length, $\mathrm{TGW}=1000$-grain weight, $\mathrm{ISW}=$ Individual spike weight, $\mathrm{HI}=$ Harvest index, GPS $=$ Grain/spike, Y= Yield

Note: Values in bold correspond to highest contribution variable.

Table 7. Summary statistics of SSRs.

\begin{tabular}{cccccc}
\hline Marker & Major allele frequency & Allele No. & Gene diversity & Heterozygosity & PIC \\
\hline Wmc332 & 0.479 & 3.000 & 0.557 & 0.021 & 0.458 \\
Wmc553 & 0.830 & 2.000 & 0.282 & 0.000 & 0.242 \\
Wm500 & 0.511 & 2.000 & 0.499 & 0.000 & 0.375 \\
Wmc474 & 0.872 & 2.000 & 0.223 & 0.000 & 0.198 \\
Wmc276 & 0.826 & 2.000 & 0.287 & 0.000 & 0.246 \\
\hline
\end{tabular}




\begin{tabular}{cccccc}
\hline Wm684 & 0.574 & 2.000 & 0.489 & 0.000 & 0.000 \\
Wmc667 & 0.622 & 2.000 & 0.470 & 0.000 & 0.360 \\
Wmc289 & 0.937 & 2.000 & 0.117 & 0.000 & 0.110 \\
Wmc570 & 0.775 & 2.000 & 0.348 & 0.000 & 0.178 \\
Wmc75 & 0.889 & 2.000 & 0.197 & 0.000 & 0.152 \\
Wmc589 & 0.909 & 2.000 & 0.165 & 0.000 & 0.344 \\
Wmc434 & 0.772 & 3.000 & 0.376 & 0.000 & 0.367 \\
Wmc477 & 0.587 & 2.000 & 0.485 & 0.000 & 0.114 \\
Wmc508 & 0.934 & 2.000 & 0.122 & 0.000 & 0.397 \\
Wmc70 & 0.708 & 3.000 & 0.448 & 0.0014 & 0.28 \\
Mean & 0.749 & 2.200 & 0.338 & & 0.000 \\
\hline
\end{tabular}

\section{DISCUSSION}

Changing environmental conditions have resulted in increased exposure of crop plants to abiotic stresses particularly high temperature. It is causing significant reduction in wheat yield throughout the world. The current research was planned to evaluate locally adapted cultivars of wheat under high temperature stress. The high yielding genotypes could be used as parents to develop new tolerant varieties. Genetic diversity is a prerequisite step for this purpose. Therefore, performance of genotypes were evaluated under field conditions followed by molecular analysis.

Varying performance of genotypes were observed under normal and high temperature stress during both years. During year 2016-17 genotypes performed better than later year, possibly due to effect of environment. Genotypes AAS-11 and K-65 performed best during $1^{\text {st }}$ year but under high temperature stress their performance reduced significantly. Whereas, Shafaq-06 and Yecora-70 performed better under high temperature stress. From these results, it may be reasoned that AAS-11 and K-65 have high potential of yield but they are susceptible to high temperature stress whereas; later genotypes Shafaq-06 and Yecora-70 are better choice under stress conditions. Tewolde et al. (2006) also reported reduced yield of certain genotypes under high temperature stress.

DTH and DPM are determinant of reproductive growth of the plants therefore these traits were more influenced by ambient temperature. Under stress, genotypes headed earlier than normal conditions. Similarly, days taken to physiological maturity were also decreased which means that high temperature reduced the growth duration of wheat crop. Negative effect of heat stress on plant developmental traits such as DTH, DPM and $\mathrm{PH}$ was also observed by some other researchers (Rehman et al., 2009; Singh et al., 2007).

Significant results revealed by ANOVA for genotype and treatment showed that the germplasm under investigation has varying responses to high temperature. Mean squares values for treatment were higher than genotypes which showed that treatment effects were more prominent than genotypic effects. Interaction between $(G$ $\mathrm{x} T)$ and $(\mathrm{G} \times \mathrm{Y})$ were also significant which showed varying performance of plant material under varying conditions. Variation in the performance of genotypes under varying climatic conditions were also observed (Degewione et al., 2013; Motamedi et al., 2012; Tadesse et al., 2012).

Higher impact of treatment (high temperature stress) and year as compared to genotype showed that studied genotypes were greatly influenced by season. Similarly, significant response to year factor also showed that genotypes responded differently under both years. Ahmad et al. (2014) showed significant impact of treatment and year on a large collection of bread wheat germplasm belonging to different origins. Sajjad et al. (2014) also reported significant difference in the performance bread wheat germplasm during different years.

Principal component analyses was used to select high performing and heat tolerant genotypes. PCA results obtained for the year 2016-17 depicted more diversity for yield related traits whereas; during 2017-18 days to physiological maturity and other plant developmental traits showed more diversity in performance. In this study we identified genotypes shafaq-06 and yecora-70 as tolerant genotypes for heat prone environments. Genotype by trait biplot grouped the genotypes using their relative performance. The study showed that during first year trait vectors for ISW, Y and GPS were in same direction which favors' that selection for any of these traits will ultimately results in increased yield. Arain et al. (2011) stated that high yielding genotypes can be selected on the bases of spike weight and number of grains per spike under stressed and non stressed environments.

Population structure analysis was performed to determine genetic relatedness among genotypes and grouped into three subpopulations which showed that germplasm is structured. Although, all the genotypes were belong to the Pakistan but presence of different subpopulation showed that the genotypes were undergone through various selection procedures to meet different breeding objectives. Interestingly all the stress tolerant genotypes cannot be grouped into same population and vice versa. These genotypes were grouped into different subpopulation which could be the result of different stress 
tolerant genes or QTLs in different genotypes (Ahmad et al., 2014; Pradhan et al., 2016).

In our study, SSR markers were employed to determine genetic diversity among wheat genotypes which have also been used extensively in wheat (Ahmad et al., 2014; Chen et al., 2012). In a study for 90 Chinese wheat genotypes, mean genetic diversity value (0.6) and average alleles/locus (5.05) were observed whereas; in current investigation average genetic diversity and alleles/locus were 0.338 and 2.2, respectively. Another study revealed a range of mean genetic diversity from 0.46 to 0.77 and major allele frequency from 4.81 to 18.1 in wheat (Huang et al., 2002; Zhang et al., 2010; Hao et al., 2011; Breseghello and Sorrells, 2006; Dreisigacker et al., 2004; Maccaferri et al., 2005).

Similarly, PIC value is a good indicator of polymorphism, which can be used for genotyping plant populations and studying genetic diversity. In current investigation, PIC value also remained very low. In a study on French wheat collection, PIC value was observed (0.66) whereas in this study only (0.33) PIC value was reported which is almost half of the French collections (Roussel et al. 2004).

In our study level of genetic diversity was low as compared to other studies because all the fifty genotypes were belong to same origin. This might be resulted in due to extensive breeding efforts using limited number of parents.

Conclusion: The wheat genotypes, AAS-11 and K-65 showed high yield under normal conditions but found sensitive to heat stress. The study identified shafaq-06 and Yecora-70 as tolerant genotypes. In comparison with previous studies, genetic diversity and PIC values in present study remained very low which suggests introduction of diverse genetic resources to the existing genetic pool. Use of biplot analysis on the basis of relative performance dataset is a suitable methodology to identify stress tolerant genotypes.

\section{REFERENCES}

Abebe, T.D., and J. Leon (2012). Spatial and temporal genetic analyses of Ethiopian barley (Hordeum vulgare L.) landraces reveal the absence of a distinct population structure. Genet. Resour. Crop Evol. 60: 1547-1558.

Ahmad, M.Q., S. H. Khan, A.S. Khan, A.Mujeeb-Kazi, and S.M. Basra (2014). Identification of QTLs for drought tolerance traits on wheat chromosome 2A using association mapping. Int. J. Agric. Biol. 16: 862-870.

Ahmad, M.Q., S.H. Khan, M. Sajjad, and I.A. Khan (2015). Analysis of drought responsive traits in hexaploid wheat (Triticum aestivum L.). Pakistan J. Agric. Sci. 52: 701-707.
Akbar, M., M. Khan, and N. Ahmad (2007). Heterosis and heterobeltiosis for improvement of wheat grain yield. J. Agri. Res. 42: 87-94.

Arain, M.A., M.A. Sial, M.A. Rajput, and A.A. Mirbahar (2011). Yield stability in bread wheat genotypes. Pakistan J. Bot. 43: 2071-2074.

Bonnett, D., M. Ellis, and X. Sirault (2008). Unravelling the effects of GA-responsive dwarfing gene Rht13 on yield and grain size. Nature. 491: 705 710.

Bonjean, A.P. and W.J. Angus. (2001). The world wheat book: a history of wheat breeding. Lavoisier Publishing, Rue Lavoisier.

Buckler, E.S., and J.M. Thornsberry (2002). Plant molecular diversity and applications to genomics. Curr. Opin. Plant Biol. 5: 107-111.

Breseghello, F., and E. Sorrells (2006). Association mapping of kernel size and milling quality in wheat (Triticum aestivum L.) cultivars. Genetics. 172: 1165-1177.

Chatrath, R., B. Mishra, G.O. Ferrara, S. Singh, and A. Joshi (2007). Challenges to wheat production in South Asia. Euphytica. 157: 447-456.

Chaturvedi, A. K., R. N. Bahuguna, D. Shah, M. Pal, and S.V.K. Jagadish (2017). High temperature stress during flowering and grain filling offsets beneficial impact of elevated $\mathrm{CO}_{2}$ on assimilate partitioning and sink-strength in rice. Sci. Rep. 7: p.8227.

Chen, X., D. Min, T.A. Yasir, and Y.G. Hu (2012). Genetic diversity, population structure and linkage disequilibrium in elite Chinese winter wheat investigated with SSR markers. PLoS One. 7: e44510.

Cossani, C.M., and M.P. Reynolds (2012). Physiological traits for improving heat tolerance in wheat. Plant Physiol. 160: 1710-1718.

Degewione, A., T. Dejene, and M. Sharif (2013). Genetic variability and traits association in bread wheat (Triticum aestivum L.) genotypes. Int. Res. J. Agric. Sci. 1: 19-29.

Dreisigacker, S., P. Zhang, M.L.Warburton, M. Van Ginkel, D. Hoisington, M.O. Bohn, and A. E. Melchinge (2004). SSR and pedigree analyses of genetic diversity among CIMMYT wheat lines targeted to different mega environments. Crop Sci. 44: 381-388.

Driedonks, N., I. Rieu, and W.H. Vriezen (2016). Breeding for plant heat tolerance at vegetative and reproductive stages. Plant Reprod. 29: 67-79.

Doyle, J.J., and J.L. Doyle (1990). Isolation of plant DNA from fresh tissue. Focus 12:13-15

Feng, B., P. Liu, G. Li, S.T. Dong, F.H. Wang, L.A. Kong, and J.W. Zhang (2014). Effect of heat stress on the photosynthetic characteristics in flag leaves at the grain-filling stage of different heat-resistant 
winter wheat varieties. J. Agron. Crop Sci. 200: 143-155.

Flint-Garcia, S.A., J.M. Thornsberry, and E.S. Buckler (2003). Structure of linkage disequilibrium in plants. Annu Rev Plant Biol. 54: 357-374.

Hao, C.Y., L.F. Wang, H.M. Ge, Y.C. Dong, and X.Y. Zhang (2011). Genetic Diversity and Linkage Disequilibrium in Chinese Bread Wheat (Triticum aestivum L.) Revealed by SSR Markers. Plos One. 6: 1-13.

Huang, Q., A. Borner, S. Roder, and W. Ganal (2002). Assessing genetic diversity of wheat (Triticum aestivum L.) germplasm using microsatellite markers. Theor. Appl. Genet. 105: 699-707.

Jiang, G.H., Y.Q. He, C.G. Xu, X.H. Li, and Q. Zhang (2004). The genetic basis of stay-green in rice analyzed in population of dihybrid lines derived from indica by japonica cross. Theor Appl Genet. 108: 688-698.

Joshi, A., B. Mishra, R. Chatrath, G.O. Ferrara, and R.P. Singh (2007). Wheat improvement in India: present status, emerging challenges and future prospects. Euphytica. 157: 431-446.

Liu, K., and S.V. Muse (2005). Power marker: an integrated analysis environment for genetic marker analysis. Bioinformatics. 21: 2128-2129.

Maccaferri, M., M.C. Sanguineti, E. Noli, and R. Tuberosa (2005). Population structure and long-range linkage disequilibrium in a durum wheat elite collection. Mol. Breed. 15: 271-289.

Motamedi, M., H.R. Naserierad, R. Naseri, and A. Soleymanifard (2012). Assessment of genotype $\times$ environment interaction for grain yield in bread wheat genotypes. Int. J. Agric. Crop Sci. 4: 13661370 .

Pradhan, S.K., S.R. Barik, A. Sahoo, S. Mohapatra, D.K. Nayak, A. Mahender, J. Meher, A. Anandan, and E. Pandit (2016). Population Structure, Genetic Diversity and Molecular Marker-Trait Association Analysis for High Temperature Stress Tolerance in rice. PLoS One. 11: p.e0160027.

Prasad, P. V. V., S.A. Staggenborg, and Z. Ristic (2008). Impacts of drought and/or heat stress on physiological, developmental, growth, and yield processes of crop plants, in Response of Crops to Limited Water: Understanding and Modeling Water Stress Effects on Plant Growth Processes, eds L. R. Ahuja, V. R. Reddy, S. A. Saseendran, and Q. Yu (Madison, WI: American Society of Agronomy), 301-355.

Pritchard, J.K., M. Stephens, and P. Donnelly (2000). Inference of population structure using multilocus genotype data. Genetics. 155: 945-959.

Rehman, A., I. Habib, N. Ahmad, M. Hussain, M. Arif Khan, J. Farooq, and M. A. Ali (2009). Screening wheat germplasm for heat tolerance at terminal growth stage. Plant Omics J. 2: 9-19.

Roussel, V., J. Koenig, M. Beckert, and F. Balfourier (2004). Molecular diversity in French bread wheat accessions related to temporal trends and breeding programmes. Theor. Appl. Genet. 108: 920-930.

Sajjad, M., S.H. Khan, M.Q. Ahmad, A. Rasheed, A. Mujeeb-Kazi, and I.A. Khan. (2014). Association Mapping Identifies QTLS on Wheat Chromosome 3A for Yield Related Traits. Cer. Res. Com. 42: 177-188.

Shi, P., Y. Zhu, L. Tang, J. Chen, T. Sun, W. Cao, and Y. Tian (2016). Differential effects of temperature and duration of heat stress during anthesis and grain filling stages in rice. Environ. Exp. Bot. 132: 28-41.

Singh, J.P., P. Shambhoo, K.N. Singh, and S. Randhir (2007). Screening of heat tolerant wheat varieties by membrane thermostability index in relation to yield and yield attributing traits. Int. J. Plant Sci. 2: $159-165$.

Steel, R.G.D., J.H. Torrie, and D.A. Dickey (1997). Principles and Procedures of Statistics: A Biological Approach. McGraw-Hill Co. New York.

Stratonovitch, P., and M.A. Semenov (2015). Heat tolerance around flowering in wheat identified as a key trait for increased yield potential in Europe under climate change. J. Exp. Bot. 66: 3599-3609.

Swan, A.R.H., and M. Sandilands (1995). Introduction to geological data analysis. Blackwell Science, Oxford. pp.446.

Tadesse, W., O. Abdalla, F. Ogbonnaya, K. Nazari, I. Tahir, and M. Baum (2012). Agronomic performance of elite stem rust resistant spring wheat genotypes and association among trial sites in the Central and West Asia and North Africa Region. Crop Sci. 52: 1105-1114.

Tilman, D., C. Balzer, J. Hill, B. L. Befort. (2011). Global food demand and the sustainable intensification of agriculture. Proceed. Nat. Acad. Sci. 108: 20260-20264.

Takumi, S., E. Nishioka, H. Morihiro, T. Kawahara, and Y. Matsuoka (2009). Natural variation of morphological traits in wild wheat progenitor Aegilops tauschii Coss. Breed Sci. 59: 579-588.

Tewolde, H., C.J. Fernandez, and C.A. Erickson (2006). Wheat cultivars adapted to post-heading high temperature stress. J. Agron. Crop Sci. 192: 111120.

Xu, W., D.T. Rosenow, and H.T. Nguyen (2000a). Staygreen trait in grain sorghum: relationship between visual rating and leaf chlorophyll concentration. Plant Breed. 119: 365-367. 
Yang, H., D. Lu, X. Shen, X. Cai, and W. Lu (2015). Heat stress at different grain filling stages affects fresh waxy maize grain yield and quality. Cereal Chem. 92: 258264.

Zhang, D.D., G.H. Bai, C.S. Zhu, J.M. Yu, and B.F. Carver (2010). Genetic Diversity, Population Structure and Linkage Disequilibrium in U.S. Elite Winter Wheat. Plant Genome. 3: 117-127.

Zhang, L., D. Liu, X. Guo, W. Yang, J. Sun, D. Wang, P. Sourdille, and A. Zhang (2011). Investigation of genetic diversity and population structure of common wheat cultivars in northern China using DArT markers. BMC Genet. 12: 42. 